\title{
Chronic Rhinosinusitis and Obstructive Sleep Apnea: CPAP Reservoir Bacterial Colonization Is Not Associated with Sinus Culture Positivity
}

\author{
Rosa B. Lipin ${ }^{1}$, Anita Deshpande ${ }^{1}$, Sarah K. Wise ${ }^{1}$, John M. DelGaudio ${ }^{1}$ and Zara M. Patel ${ }^{2, *}$ \\ 1 Department of Otolaryngology—Head and Neck Surgery, Emory University School of Medicine, Atlanta, \\ GA 30308, USA; rosa.bene.lipin@emory.edu (R.B.L.); asdeshp@emory.edu (A.D.); \\ skmille@emory.edu (S.K.W.); jdelgau@emory.edu (J.M.D.) \\ 2 Department of Otolaryngology-Head and Neck Surgery, Stanford University School of Medicine, Stanford, \\ CA 94305, USA \\ * Correspondence: zpatel@ohns.stanford.edu; Tel.: +1-650-724-1745; Fax: +1-650-725-8502
}

Academic Editors: Claudina A. Pérez Novo and César Picado

Received: 24 December 2015; Accepted: 28 February 2016; Published: 9 March 2016

\begin{abstract}
Chronic rhinosinusitis (CRS) and obstructive sleep apnea (OSA) are both highly prevalent chronic diseases in the United States. Association between culture positivity of CPAP machines and sinus samples has not been studied in patients with both disease states. Our objective was to compare the microbes present in the sinus cavities and CPAP reservoirs of patients with both CRS and OSA. Patients from an academic tertiary care Rhinology practice were identified with both CRS and OSA and enrolled prospectively. Inclusion criteria included age over 18 years; diagnosis of OSA by sleep study; regular CPAP use; and an active diagnosis of CRS. Exclusion criteria included treatment with antibiotics or cleaning of the CPAP reservoir in the month prior. Cultures were taken from participants' sinus cavities and CPAP reservoirs and resulting microbial growth was compared. The most common organisms on CPAP culture were Enterobacter cloacae and Acinetobacter baumanii, whereas the most common on sinus culture were Staphyloccoccus aureus and Pseudomonas aeruginosa. Microbial growth from the sinus cavities and the CPAP reservoirs were not concordant in any of our patients. There is no association between bacterial colonization of the CPAP reservoir and the sinus cavities of those with CRS and OSA based on microbiologic cultures.
\end{abstract}

Keywords: chronic rhinosinusitis (CRS); obstructive sleep apnea (OSA); continuous positive airway pressure (CPAP); unified upper airway; bacterial colonization; microbial culture

\section{Introduction}

Chronic rhinosinusitis (CRS) is an end result of over 12 weeks of symptoms from underlying inflammation of the lining of the paranasal cavities, leading to over 18.3 million office visits a year [1]. Patients with CRS see primary care providers twice as much as those without the disease, and are five times more likely to be prescribed antibiotics [2]. The presentation of this disease can be highly variable, and the etiology remains poorly understood. The pathophysiology is likely multi-factorial, involving infectious agents, innate and adaptive immune responses, and other host and environmental factors [3]. As there is currently no standardized therapy for CRS, patients are managed using a combination of strategies, including systemic and topical corticosteroids, antimicrobial agents, intranasal saline, immune modulators, and endoscopic sinus surgery [4].

Obstructive sleep apnea (OSA) is another common chronic disease, found in $9 \%-26 \%$ of middle aged adults and characterized by the presence of five or more episodes of complete (apnea) or partial (hypopnea) airflow obstruction per hour while asleep [5]. Untreated OSA is thought to contribute 
\$3.4 billion annually to U.S. health care costs [6]. The gold standard treatment for this condition is continuous positive airway pressure (CPAP), which functions to splint the upper airway open during both inspiration and expiration, and thus counteracts the structural airway abnormalities leading to collapse during sleep [5]. Unfortunately, the use of CPAP may lead to upper airway dryness and nasal congestion, side effects which may compromise patient compliance and satisfaction [7]. Heated humidification has been shown to yield higher compliance, increased satisfaction, feeling more refreshed on awakening, and greater comfort as opposed to using CPAP without humidity $[7,8]$. However, Sanner et al. found that users of CPAP with humidification experienced an increased rate of upper airway infection compared to OSA patients utilizing non-CPAP therapies, with a significantly higher rate observed in patients who cleaned their devices inadequately [9].

The association between OSA and CRS has not been extensively studied. Chin $e t$ al. administered the chronic sinusitis survey (CSS) to OSA patients who were regular CPAP users and analyzed the relationship between CPAP reservoir culture positivity and CRS symptomatology based on CSS scores, finding no association between the two [10]. Of note, this group excluded patients with a known history of CRS, thus leaving a gap in our knowledge about how CPAP culture positivity may affect this subgroup of patients.

To our knowledge, no studies to date have examined the association between microbes present in the sinonasal cavities and CPAP machine reservoirs of patients with known diagnoses of both CRS and OSA. In this study, we first set out to establish the rate of CPAP reservoir culture positivity in patients with CRS. In addition, we aimed to compare bacterial culture results from sinonasal cavities and CPAP reservoirs of patients with both OSA and CRS to determine if an association existed.

\section{Materials and Methods}

After approval was obtained from the Institutional Review Board, study participants were identified at an academic tertiary care Rhinology practice using ICD-9 codes for obstructive sleep apnea and chronic rhinosinusitis. Patients were either contacted on the phone or met with a research assistant in clinic to determine if they met the inclusion criteria: age over 18 years, diagnosis of OSA by polysomnography, regular CPAP use (meaning use of the machine all night, every night and waking up with the machine still on and attached in correct postion), and an active diagnosis of CRS (not symptomatically controlled, with objective evidence via CT and/or nasal endoscopy of CRS). Exclusion criteria were: treatment with antibiotics or cleaning of the CPAP reservoir in the month prior to enrollment. If participants met criteria and were interested in participation they were prospectively enrolled in the study, gave written informed consent, and had cultures taken from their sinonasal cavities. If the patients had not been operated on in the past, the culture was taken from the middle meatus. If they had been operated on, a culture was taken directly from the diseased sinus. If participants were able to bring their CPAP reservoirs in, cultures were taken from the hydration chambers of the machines at that time as well. Otherwise, patients were given a culture swab with instructions on how to culture their CPAP reservoirs and return the swab for laboratory testing.

Both sets of swabs from each patient were cultured and results of speciation were recorded. Swabs were transported in Amies gel medium, appropriate for both aerobic and anaerobic growth. Resulting microbial growth from sinonasal cavity samples for each patient were compared to microbial growth from that individual's CPAP reservoir. Cultures were reported in a standard 1+ through 4+ fashion based on the common practice of cultures being inoculated onto media using a sterile loop that sequentially dilutes the specimen from the first area or quadrant of the medium to the last area or quadrant. Results are then reported depending on which areas or quadrants demonstrate bacterial growth with $4+$ showing bacterial growth even with the most dilute concentration of specimen. Specimens were processed on the day of submission. 


\section{Results}

Ten patients meeting inclusion criteria were enrolled in the study and nine had both sinonasal and CPAP reservoir samples obtained. Of these nine patients remaining at the conclusion of the study, seven (78\%) were male and two (22\%) were female. Mean age was 52 (range, 39-68 years), and four $(44 \%)$ had undergone endoscopic sinus surgery in the past. All patients had undergone multiple courses of antibiotics previously (although none in the month leading up to the culture), including amoxicillin, amoxicillin with clavulanate, levofloxacin, ciprofloxacin, cephalexin, and doxycycline. All patients who had previously undergone sinus surgery had already been on CPAP prior to that intervention and continued to use it after the surgery.

The final culture results for the nine patients with both sets of samples taken are listed in Table 1. All nine CPAP reservoir samples displayed culture positivity. The most common organisms on CPAP culture were Enterobacter cloacae, Acinetobacter baumanii, Candida parapsilosis, and Brevidimonas diminuta. All sinus cultures were also positive, but with differing organisms. The most common on sinus culture were Staphyloccoccus aureus, Staphlococcus epidermidis, and Pseudomonas aeruginosa. Speciation of microbial growth from sinonasal samples was not associated with CPAP reservoir growth in any of the nine study participants.

Table 1. CPAP reservoir and sinonasal culture results.

\begin{tabular}{|c|c|c|}
\hline Patient & CPAP Reservoir Culture & Sinonasal Sample \\
\hline 1 & $\begin{array}{c}\text { 1+ Enterobacter cloacae } \\
\text { 3+ Burkholderia cepacia complex } \\
\text { 1+ Staphylococcus coagulase negative } \\
\text { 1+ Candida parapsilosis } \\
1+\text { Rhodotorula mucilaginosa }\end{array}$ & 1+ Staphylococcus aureus \\
\hline 2 & $\begin{array}{c}2+\text { Enterobacter cloacae } \\
2+\text { Brevundimonas diminuta }\end{array}$ & $\begin{array}{c}\text { 2+ Staphylococcus aureus } \\
\text { 1+ Staphylococcus epidermidis }\end{array}$ \\
\hline 3 & $\begin{array}{l}\text { 1+ Trichosporon asahii } \\
1+\text { Serratia marascens }\end{array}$ & 4+ Pseudomonas \\
\hline 4 & 1+ Acinetobacter baumanii & 2+ Staphylococcus aureus \\
\hline 5 & $2+$ Acinetobacter baumanii & $\begin{array}{c}2+\text { Staphylococcus aureus } \\
1+\text { Staphylococcus epidermidis }\end{array}$ \\
\hline 6 & $2+$ Candida parapsilosis & $\begin{array}{c}2+\text { Pseudomonas } \\
1+\text { Staphylococcus epidermidis }\end{array}$ \\
\hline 7 & $\begin{array}{c}2+\text { Enterobacter cloacae } \\
1+\text { Brevundimonas diminuta }\end{array}$ & $2+$ Citrobacter koseri \\
\hline 8 & $\begin{array}{c}2+\text { Enterobacter cloacae } \\
1+\text { Acinetobacter baumanii }\end{array}$ & 2+ Pneuтососсиs \\
\hline 9 & 1+Enterobacter cloacae & $\begin{array}{c}2+\text { Staphylococcus aureus } \\
1+\text { Pseudomonas }\end{array}$ \\
\hline
\end{tabular}

\section{Discussion}

Though cultures of all CPAP machine reservoirs tested in our study produced bacterial growth, we found no association between bacterial colonization of the CPAP reservoir and the sinus cavities of patients with both CRS and OSA. This finding reflects that observed by Chin et al. with one major exception. Whereas Chin et al. found culture positivity in only $48.6 \%$ of CPAP reservoirs, we witnessed this phenomenon in $100 \%$ of our samples, albeit our sample size was small [10]. This finding lends evidence to the theory that the warm, moist environment of CPAP machine reservoirs is conducive to bacterial growth, but this microbial proliferation does not appear to have clinical relevance in relation to CRS. 
In our study, the three most commonly observed microorganisms on sinus culture were Staphyloccoccus aureus, Staphlococcus epidermidis, and Pseudomonas aeruginosa. This finding is concordant with prior studies showing that the most common bacterial isolates in CRS patients undergoing endoscopic sinus surgery are coagulase-negative Staphlococcus, gram-negative rods, and Staphylococcus aureus [11,12]. Chin et al. found that the most commonly cultured organisms from CPAP reservoirs were gram-negative rods [10]. Consistent with the Chin study, three of the four most commonly cultured organisms from our study participants' CPAP reservoir samples (Enterobacter cloacae, Acinetobacter baumanii, and Brevidimonas diminuta) are classified as gram-negative rods.

The small sample size of our study is an important limitation. Although we cannot make any statistically significant conclusions regarding our data and results, our findings are certainly interesting, as no patient displayed any association between microbial growth cultured from their sinonasal samples and CPAP reservoirs. Our observations are similar to those of Lee et al., who found that culture positivity of nasal irrigation bottle and fluid was not related to higher infection rates following endoscopic sinus surgery [13].

Another important limitation of this study stems from the growing knowledge we have of the sinus microbiome. Recent studies have shown a vast and diverse array of bacteria living within both normal and diseased sinuses, with many of these organisms not regularly identified on microbial culture [14-16]. Whether or not the bacteria found in the CPAP cultures may have joined this larger, less readily measurable population is unknown. The cost of PCR and other advanced techniques has generally limited full examination of the microbiome to research environments, and clinicians still rely heavily on culture results to drive therapy. In this study we chose to limit our investigation to the tool most commonly used clinically to search for bacterial growth, but certainly further study using those other tools may demonstrate more subtle additions or changes to the microbial environment of the sinuses in these patients.

Although we did not observe a direct association between microbial growth in CPAP reservoirs and sinonasal samples, proper care and cleaning of CPAP reservoirs should be emphasized to patients diagnosed with OSA, as prior evidence has shown that this population experiences an increased rate of upper airway infections [10]. As the development and perpetuation of CRS is still poorly understood, it may be possible that microbial growth from CPAP machines still plays a role in the etiology of CRS, perhaps by altering innate and/or adaptive immune response prior to the development of CRS. Additionally, given the subtypes of CRS and their varied responses to different therapies, we support the continued investigation of infectious sources for CRS symptoms, including culturing sinonasal samples and tailoring antibiotic choice to speciation and sensitivity results.

To our knowledge, this is the first study to examine the association between microbes present in the sinus cavities and CPAP machine reservoirs of patients with known diagnoses of both CRS and OSA. We found that culture results from both sinonasal and CPAP reservoir samples reflected data published in the literature about which microbes are most typical in these settings, lending credibility to our study despite its small sample size. We conclude it is unlikely, in OSA patients suffering CRS symptoms, that the bacteria growing in their CPAP machine is leading to the development or perpetuation of their CRS.

\section{Conclusions}

We found no association between microbial culture of the CPAP reservoirs and the sinus cavities of patients with dual diagnoses of CRS and OSA. Further study looking more deeply into the microbiome with enhanced tools for measuring bacterial growth may further our knowledge of this subset of patients.

Acknowledgments: Rosa Lipin: No financial disclosures; Anita Deshpande: No financial disclosures; Sarah Wise: Greer Laboratories-Scientific Advisory Board, Genentech, Research funding; John DelGaudio: No financial disclosures; Zara Patel: No financial disclosures. 
Author Contributions: Z.M.P. and R.B.L. conceived and designed the study; R.B.L. and A.D. performed the study; Z.M.P., R.B.L. and A.D. analyzed the data; S.K.W. and J.M.D. contributed patients and played a major role in editing of the manuscript; Z.M.P. wrote the manuscript; R.B.L., Z.M.P., A.D., S.K.W. and J.M.D. all contributed to revising and editing of the manuscript.

Conflicts of Interest: The authors declare no conflict of interest.

\section{References}

1. Rosenfeld, R.M.; Piccirillo, J.F.; Chandrasekhar, S.S.; Brook, I.; Kumar, K.A.; Kramper, M.; Orlandi, R.R.; Palmer, J.N.; Patel, Z.M.; Peters, A.; et al. Clinical practice guideline (update) adult sinusitis. Otolaryngol. Head Neck Surg. 2015, 152, 1-39. [CrossRef] [PubMed]

2. Ray, N.; Baraniuk, J.; Thamer, M. Healthcare expenditures for sinusitis in 1996: Contributions of asthma, rhinitis, and other airway disorders. J. Allergy Clin. Immunol. 1999, 103, 408-414. [CrossRef]

3. Manes, R.; Bhatra, P. Etiology, diagnosis and management of chronic rhinosinusitis. Expert Rev. Anti. Infect. Ther. 2013, 11, 25-35. [CrossRef] [PubMed]

4. Cain, R.; Lal, D. Update on the management of chronic rhinosinusitis. Infect. Drug Resist. 2013, 6, 1-14. [PubMed]

5. Basner, R. Continuous positive airway pressure for obstructive sleep apnea. N. Engl. J. Med. 2007, 17, 1751-1758. [CrossRef] [PubMed]

6. Kapur, V.; Blough, D.K.; Sandblom, R.E.; Hert, R.; de Maine, J.B.; Sullivan, S.D.; Psaty, B.M. The medical cost of undiagnosed sleep apnea. Sleep 1999, 22, 749-755. [PubMed]

7. Massie, C.A.; Hart, R.W.; Peralez, K.; Richards, G.N. Effects of humidification on nasal symptoms and compliance in sleep apnea patients using continuous positive airway pressure. Chest 1999, 116, 403-408. [CrossRef] [PubMed]

8. Wiest, G.H.; Lehnert, G.; Brûck, W.M.; Meyer, M.; Hahn, E.G.; Ficker, J.H. A heated humidifier reduces upper airway dryness during continuous positive airway pressure therapy. Respir. Med. 1999, 93, 21-26. [CrossRef]

9. Sanner, B.M.; Fluerenbrock, N.; Kleiber-Imbeck, A.; Mueller, J.B.; Zidek, W. Effect of continuous positive airway pressure therapy on infectious complication in patients with obstructive sleep apnea syndrome. Respiration 2001, 68, 483-487. [CrossRef] [PubMed]

10. Chin, C.J.; George, C.; Lannigan, R.; Rotenberg, B.W. Association CPAP bacterial colonization with chronic rhinosinusitis. J. Clin. Sleep Med. 2013, 9, 747-750. [CrossRef] [PubMed]

11. Nadel, D.; Lanza, D.; Kennedy, D. Endoscopically guided cultures in chronic sinusitis. Am. J. Rhinol. 1998, 12, 233-241. [CrossRef] [PubMed]

12. Kingdom, T.; Swain, R. The microbiology and antimicrobial resistancepatterns in chronic rhinosinusitis. Am. J. Otolaryngol. 2004, 25, 323-328. [CrossRef] [PubMed]

13. Lee, J.M.; Nayak, J.V.; Doghramji, L.L.; Welch, K.C.; Chiu, A.G. Assessing the risk of irrigation bottle and fluid contamination after endoscopic sinus surgery. Am. J. Rhinol. Allergy 2010, 24, 197-199. [CrossRef] [PubMed]

14. Ramakrishnan, V.R.; Feazel, L.M.; Gitomer, S.A.; Ir, D.; Robertson, C.E.; Frank, D.N. The microbiome of the middle meatus in healthy adults. PLoS ONE 2013, 8, e85507. [CrossRef] [PubMed]

15. Hauser, L.J.; Feazel, L.M.; Ir, D.; Fang, R.; Wagner, B.D.; Robertson, C.E.; Frank, D.N.; Ramakrishnan, V.R. Sinus culture poorly predicts resident microbiota. Int. Forum Allergy Rhinol. 2015, 5, 3-9. [CrossRef] [PubMed]

16. Ramakrishnan, V.R.; Hauser, L.J.; Feazel, L.M.; Ir, D.; Robertson, C.E.; Frank, D.N. Sinus microbiota varies among chronic rhinosinusitis phenotypes and predicts surgical outcomes. J. Allergy Clin. Immunol. 2015, 136, 334-342. [PubMed]

(C) 2016 by the authors; licensee MDPI, Basel, Switzerland. This article is an open access article distributed under the terms and conditions of the Creative Commons by Attribution (CC-BY) license (http://creativecommons.org/licenses/by/4.0/). 